\title{
DNA Polymerase Recognition of Unnatural Base Pair: 1-Methoxy-3-methylbenzene and 6-Methyl-1(2H)-isoquinolinone
}

\author{
Kyoung-Ae Kim and Gil Tae Hwang* \\ Department of Chemistry, Kyungpook National University, Daegu 702-701, Korea. "E-mail: giltae@knu.ac.kr \\ Received July 28, 2010, Accepted August 3, 2010
}

Key Words: DNA, Nucleobase, Base pair, Unnatural nucleobase, DNA replication

The faithful replication of DNA by DNA polymerases is necessary for the expansion of both the genetic alphabet and the genetic code. ${ }^{1}$ The stability and sequence specificity of a natural DNA duplex are based on the complementary and orthogonal Watson-Crick hydrogen-bonding patterns of $\mathrm{dA}: \mathrm{dT}$ and $\mathrm{dC}: \mathrm{dG}$ base pairs. Although inter-nucleobase hydrogen bonding and shape complementarity are important for the stability and faithful copying of DNA, there is no reason to assume that the pairing of two unnatural bases could not be driven by other intermolecular interactions, for example, those based on hydrophobic interactions and van der Waals forces. Some groups have used this concept to explore the development of unnatural nucleotides bearing nucleobase analogs that pair via hydrophobic interactions and packing forces. ${ }^{2-5}$ Recently, Romesberg's group found that the heteropair formed between 1-methoxy-3-methylbenzene $\mathbf{1}$ and 6-methyl-1 $(2 H)$-isoquinolinethione $\mathbf{2}$ is a very promising, predominantly hydrophobic base pair. This base pair is synthesized by the insertion of one unnatural triphosphate opposite the other unnatural nucleotide in the template, and it is then extended by the insertion of the next correct dNTP (natural triphosphates), with relatively high efficiency and fidelity, by diverse DNA polymerases (Figure 1). ${ }^{5}$ However, the slowest step in the replication of DNA containing this heteropair is the incorporation of $\mathbf{1}$ triphosphate opposite $\mathbf{2}$ in the template. Also, $\mathrm{dG}$ inserts competitively opposite $\mathbf{2}$ in the template, which may limit the use of this heteropair in biological and biotechnological applications. We are therefore interested in the modifications of $\mathbf{1}$ or $\mathbf{2}$ to optimize the formation of unnatural heteropairs. Herein, we report the DNA replication results for two nucleotides, 6-methyl-1 $(2 H)$-isoquinolinone $\mathbf{3}$, in which an oxygen atom is substituted for the sulfur atom in $\mathbf{2}$ to compare the effect of the oxygen atom on replication properties, and 7-methyl-1(2H)isoquinolinone 4 , in which, as well as replacing the sulfur atom in $\mathbf{2}$ by an oxygen atom, the methyl substituent is moved from position 6 to position 7. The substitution at position 7 is expected to bury the methyl group within the duplex, as shown

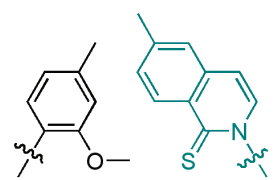

$1: 2$

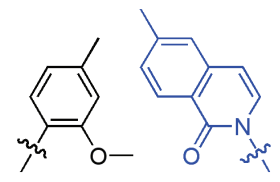

$1: 3$

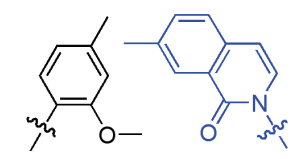

$1: 4$
Figure 1. Unnatural nucleotides used in current study. Sugars and phosphates have been omitted for clarity. in Figure 1.

In general, faithful polymerase-mediated synthesis can be considered to occur in two well-defined steps. ${ }^{6}$ First, the selective incorporation of the correct triphosphate, which must be more efficient than the misincorporations of incorrect triphosphates opposite a template nucleotide. Second, DNA extension following the synthesis of the pair, which must be faster when the pair is correct than when it is incorrect. In this work, the correct pairs being considered as new genetic alphabet candidates are 1:3 and 1:4; the misincorporations of dNTPs opposite 1, 3, or 4 in the template and the self-pair synthesis of 3 or $\mathbf{4}$ (3:3 or $4: 4$ formation) and their extensions therefore need to be much slower than the rates of synthesis of the correct heteropairs and their extensions. Each unnatural and natural base was incorporated into the primer-template substrates shown in Table 1. The efficiency and selectivity of unnatural DNA synthesis by the exonuclease deficient Klenow fragment of E. coli DNA polymerase $\mathrm{I}(\mathrm{Kf})^{7}$ were examined in the context of both correct pair and incorrect pair syntheses and their extensions using a single nucleotide incorporation assay. This gave the secondorder rate constant $k_{\text {cat }} / K_{\mathrm{M}}$ (or efficiency). The lower limit of detection in this study is $k_{\text {cat }} / K_{\mathrm{M}} \sim 1.0 \times 10^{3} \mathrm{M}^{-1} \mathrm{~min}^{-1}$. Throughout, heteropairs (natural or unnatural) are denoted as $\mathrm{dX}: \mathrm{dY}$, with $\mathrm{dX}$ indicating the nucleotide in the primer strand and $\mathrm{dY}$ indicating the nucleotide in the template strand.

We first examined the Kf-mediated incorporation of correct and incorrect triphosphates opposite $\mathbf{3}$ and $\mathbf{4}$ in the templates. For reference, we used the steady-state rates of dA:dT and dG:dT as natural base pairs and $\mathrm{dX}: \mathbf{2}$ (where $\mathrm{X}$ denotes a natural or unnatural base) as unnatural base pairs (Table 1, entries 1, 2, and 7-12). ${ }^{5}$ To address the effects of substituting oxygen for sulfur in the isoquinolinethione scaffold, we characterized the Kf-mediated synthesis of the isoquinolinone ( 3 and $\mathbf{4}$ ) self-pairs and mispairs with dNTPs (Table 1, entries 14-18 and 20-24). All the misincorporations of dNTPs opposite $\mathbf{3}$ and $\mathbf{4}$ in the templates were inefficient, with second-order rate constants $\left(k_{\text {cat }} / K_{\mathrm{M}}\right)$ varying between $<1.0 \times 10^{3} \mathrm{M}^{-1} \mathrm{~min}^{-1}$ and $2.7 \times 10^{3}$ $\mathrm{M}^{-1} \mathrm{~min}^{-1}$. Interestingly, both 3 and $\mathbf{4}$ templated the insertion of dGTP with efficiencies of $1.5 \times 10^{3} \mathrm{M}^{-1} \mathrm{~min}^{-1}$ and $2.5 \times 10^{3} \mathrm{M}^{-1}$ $\mathrm{min}^{-1}$, respectively; dGTP was inserted opposite 2 with a rate of $1.3 \times 10^{5} \mathrm{M}^{-1} \mathrm{~min}^{-1}$, which was the most efficient mispairing of 2. Thus, oxygen substitution was significantly less favorable for pairing with all natural triphosphates. Next, we characterized the steady-state rates for the Kf-mediated synthesis of the isoquinolinone-based nucleotide self-pairs (Table 1, entries 14 
Table 1. Incorporation of $\mathrm{d} \mathbf{X}$ triphosphates opposite unnatural bases in the template ${ }^{a}$

\begin{tabular}{|c|c|c|c|c|c|}
\hline entry & X & $\mathbf{Y}$ & $\underset{\left(\mathrm{min}^{-1}\right)}{k_{\text {cat }}}$ & $\begin{array}{c}K_{\mathrm{M}} \\
(\mu \mathrm{M})\end{array}$ & $\begin{array}{c}k_{\text {cat }} / K_{\mathrm{M}} \\
\left(\mathrm{M}^{-1} \min ^{-1}\right)\end{array}$ \\
\hline $1^{b}$ & $\mathrm{dT}$ & dA & $6.0 \pm 0.9$ & $0.019 \pm 0.001$ & $3.2 \times 10^{8}$ \\
\hline $2^{b}$ & $\mathrm{dT}$ & $\mathrm{dG}$ & $4.4 \pm 0.3$ & $77 \pm 12$ & $5.7 \times 10^{4}$ \\
\hline $3^{b}$ & 1 & 1 & $5.1 \pm 0.7$ & $44 \pm 4$ & $1.2 \times 10^{5}$ \\
\hline $4^{b}$ & 2 & 1 & $8.5 \pm 0.1$ & $0.18 \pm 0.01$ & $4.7 \times 10^{7}$ \\
\hline 5 & 3 & 1 & $25 \pm 2$ & $12 \pm 1$ & $2.1 \times 10^{6}$ \\
\hline $6^{b}$ & 4 & 1 & $10 \pm 2$ & $51 \pm 5$ & $2.0 \times 10^{5}$ \\
\hline $7^{b}$ & 1 & 2 & $12 \pm 1$ & $33 \pm 6$ & $3.6 \times 10^{5}$ \\
\hline $8^{b}$ & 2 & 2 & $1.7 \pm 0.5$ & $63 \pm 4$ & $2.7 \times 10^{4}$ \\
\hline $9^{b}$ & $\mathrm{dA}$ & 2 & $1.2 \pm 0.3$ & $54 \pm 6$ & $2.2 \times 10^{4}$ \\
\hline $10^{b}$ & $\mathrm{dC}$ & 2 & $\mathrm{nd}^{c}$ & $\mathrm{nd}^{c}$ & $<1.0 \times 10^{3}$ \\
\hline $11^{b}$ & $\mathrm{dG}$ & 2 & $4.6 \pm 0.6$ & $36 \pm 4$ & $1.3 \times 10^{5}$ \\
\hline $12^{b}$ & $\mathrm{dT}$ & 2 & $1.7 \pm 0.2$ & $130 \pm 19$ & $1.3 \times 10^{4}$ \\
\hline 13 & 1 & 3 & $1.7 \pm 0.1$ & $153 \pm 8$ & $1.1 \times 10^{4}$ \\
\hline 14 & 3 & 3 & $0.16 \pm 0.05$ & $105 \pm 29$ & $1.5 \times 10^{3}$ \\
\hline 15 & dA & 3 & $0.07 \pm 0.01$ & $46 \pm 6$ & $1.4 \times 10^{3}$ \\
\hline 16 & $\mathrm{dC}$ & 3 & $\mathrm{nd}^{c}$ & $\mathrm{nd}^{c}$ & $<1.0 \times 10^{3}$ \\
\hline 17 & $\mathrm{dG}$ & 3 & $0.20 \pm 0.06$ & $131 \pm 8$ & $1.5 \times 10^{3}$ \\
\hline 18 & dT & 3 & $0.37 \pm 0.07$ & $137 \pm 24$ & $2.7 \times 10^{3}$ \\
\hline 19 & 1 & 4 & $0.13 \pm 0.01$ & $44 \pm 10$ & $3.0 \times 10^{3}$ \\
\hline 20 & 4 & 4 & $0.41 \pm 0.14$ & $27 \pm 6$ & $1.5 \times 10^{4}$ \\
\hline 21 & $\mathrm{dA}$ & 4 & $\mathrm{nd}^{c}$ & $\mathrm{nd}^{c}$ & $<1.0 \times 10^{3}$ \\
\hline 22 & $\mathrm{dC}$ & 4 & $\mathrm{nd}^{c}$ & $\mathrm{nd}^{c}$ & $<1.0 \times 10^{3}$ \\
\hline 23 & $\mathrm{dG}$ & 4 & $0.24 \pm 0.01$ & $97 \pm 10$ & $2.5 \times 10^{3}$ \\
\hline 24 & $\mathrm{dT}$ & 4 & $\mathrm{nd}^{c}$ & $\mathrm{nd}^{c}$ & $<1.0 \times 10^{3}$ \\
\hline
\end{tabular}

${ }^{a}$ See the Experimental Section for details. ${ }^{b}$ References $5 .{ }^{c}$ Reaction was too inefficient for $k_{\mathrm{cat}}$ and $K_{\mathrm{M}}$ to be determined. Error reported is standard deviation calculated from three independent experiments.

and 20). The self-pair 4:4 was synthesized with a rate of $1.5 \times$ $10^{4} \mathrm{M}^{-1} \mathrm{~min}^{-1}$. This rate was similar to the $2: 2$ self-pair synthesis rate (Table 1, entry 8 ). The synthesis of self-pair 3:3 was about 10 times less efficient than those of self-pairs $2: 2$ and $\mathbf{4 : 4}$. This demonstrates that simple oxygen substitution can substantially decrease the rates of self-pair synthesis.

To examine the efficiency of unnatural base pairs, we first measured the rates at which each unnatural triphosphate was inserted opposite a correct nucleotide $\mathbf{3}$ or $\mathbf{4}$ in the template. The $\mathbf{3}$ and $\mathbf{4}$ triphosphates were inserted opposite $\mathbf{1}$ to yield heteropairs 3:1 and 4:1 with efficiencies of $2.1 \times 10^{6} \mathrm{M}^{-1} \mathrm{~min}^{-1}$ and $2.0 \times 10^{5} \mathrm{M}^{-1} \mathrm{~min}^{-1}$, respectively, i.e., 22 and 240 times less efficiently than the insertion of parent 2 (Table 1, entries 5 and 6). The heteropair 1:4 (primer:template) was synthesized at a rate of $3.0 \times 10^{3} \mathrm{M}^{-1} \mathrm{~min}^{-1}$ (Table 1, entry 19). Overall, unnatural base pair syntheses with $\mathbf{1}$ and $\mathbf{4}$ were inefficient, and they had rates competitive with those of self-pairs 1:1 and 4:4 (Table 1, entries 3 and 20). Thus, within the isoquinolinone scaffold, the methyl group at the 7-position had a negative effect on unnatural base pair synthesis. $\mathbf{1}$ was inserted opposite $\mathbf{3}$ with an efficiency
Table 2. Rates of correct extension of unnatural base pairs ${ }^{a}$

5'-d(TAATACGACTCACTATAGGGAGAX)

3'-d(ATTATGCTGAGTGATATCCCTCTYGCTAGGTTACGGCA GGATCGC)

\begin{tabular}{cccccc}
\hline entry & $\mathrm{X}$ & $\mathbf{Y}$ & $\begin{array}{c}k_{\mathrm{cat}} \\
\left(\mathrm{min}^{-1}\right)\end{array}$ & $\begin{array}{c}K_{\mathrm{M}} \\
(\mu \mathrm{M})\end{array}$ & $\begin{array}{c}k_{\text {cat }} / K_{\mathrm{M}} \\
\left(\mathrm{M}^{-1} \mathrm{~min}^{-1}\right)\end{array}$ \\
\hline $1^{b}$ & $\mathrm{dT}$ & $\mathrm{dA}$ & $1.3 \pm 0.4$ & $0.0077 \pm 0.0044$ & $1.7 \times 10^{8}$ \\
$2^{b}$ & $\mathrm{dT}$ & $\mathrm{dG}$ & $3.6 \pm 0.5$ & $7.5 \pm 1.6$ & $4.8 \times 10^{5}$ \\
$3^{b}$ & $\mathbf{2}$ & $\mathbf{1}$ & $3.8 \pm 0.3$ & $5.7 \pm 1.2$ & $6.7 \times 10^{5}$ \\
4 & $\mathbf{3}$ & $\mathbf{1}$ & $15 \pm 3$ & $8.2 \pm 2.3$ & $1.8 \times 10^{6}$ \\
$5^{b}$ & $\mathbf{1}$ & $\mathbf{2}$ & $6.4 \pm 1.1$ & $3.4 \pm 0.3$ & $1.9 \times 10^{6}$ \\
6 & $\mathbf{1}$ & $\mathbf{3}$ & $0.85 \pm 0.08$ & $208 \pm 24$ & $4.1 \times 10^{3}$ \\
\hline
\end{tabular}

${ }^{a}$ See the Experimental Section for details. ${ }^{b}$ References 5. ${ }^{c}$ Reaction was too inefficient for $k_{\text {cat }}$ and $K_{\mathrm{M}}$ to be determined. Error reported is standard deviation calculated from three independent experiments.

of $1.1 \times 10^{4} \mathrm{M}^{-1} \mathrm{~min}^{-1}$ (Table 1, entry 13), which was 33 times less efficient than the insertion opposite 2 . Although the heteropair synthesis rates between $\mathbf{1}$ and $\mathbf{3}$ in both directions were less efficient than those between $\mathbf{1}$ and $\mathbf{2}$, the synthesis of 1:3 heteropairs was 4.1 times more efficient than dT:3 mispair synthesis (Table 1, entry 18), which was the most competitive mispair with $\mathbf{3}$ in the template. Furthermore, the insertion of $\mathbf{3}$ triphosphate opposite $\mathbf{1}$ in the template was 18 times more efficient than the insertion of $\mathbf{1}$ triphosphate to form a self-pair (entry 3 ), and it was 21 times more efficient than dATP insertion, giving the most competitive mispair (data not shown, $k_{\text {cat }} / K_{\mathrm{M}}=$ $1.0 \times 10^{5} \mathrm{M}^{-1} \mathrm{~min}^{-1}$ ).

dGTP was inserted opposite 2 with an efficiency of $1.3 \times 10^{5}$ $\mathrm{M}^{-1} \min ^{-1}$ (Table 1, entry 11 ), which was only 2.8 times less efficient than the insertion of $\mathbf{1}$ triphosphate opposite 2 . Thus, 2 directs the insertion of $\mathrm{dG}$ triphosphate relatively efficiently, which is problematic in terms of fidelity. We then examined the extension of the unnatural base pair by the insertion of dCTP opposite a template $\mathrm{dG}$ because the preferential extension of a correct primer terminus is also critical for the high fidelity replication of DNA. Kf extended the dA:dT pair and the dG:dT mispair with second-order rate constants of $1.7 \times 10^{8} \mathrm{M}^{-1} \mathrm{~min}^{-1}$ and $4.8 \times 10^{5} \mathrm{M}^{-1} \mathrm{~min}^{-1}$, respectively, resulting in a fidelity in this context of 350 (Table 2, entries 1 and 2). ${ }^{5}$ The parent heteropairs 2:1 and 1:2 were extended with second-order rate constants of $6.7 \times 10^{5} \mathrm{M}^{-1} \mathrm{~min}^{-1}$ and $1.9 \times 10^{6} \mathrm{M}^{-1} \mathrm{~min}^{-1}$, respectively (Table 2, entries 3 and 5). ${ }^{5}$ Interestingly, the extensions of the two heteropairs 1:3 and 3:1 differ significantly (Table 2, entries 4 and 6). The extension of the heteropair $1: 3$ was 460 times slower than the extension of the heteropair 1:2, but the heteropair 3:1 was extended with an efficiency of $1.8 \times 10^{6} \mathrm{M}^{-1}$ $\mathrm{min}^{-1}$, which was actually more efficient than the corresponding heteropair 2:1. This result demonstrated that the extensions of unnatural primer termini differed significantly from each other.

$\mathrm{Kf}$ synthesized the heteropair relatively efficiently in either strand context; the insertion of $\mathbf{2}$ opposite $\mathbf{1}$ was significantly more efficient than the synthesis of the heteropair by the insertion of $\mathbf{1}$ opposite $\mathbf{2}$, but their extension rates were similar. This type of polar recognition by $\mathrm{Kf}$ of the $\mathbf{1 : 3}$ heteropair was ob- 
served in both synthesis and extension. Thus, the most troublesome aspect of DNA replication of the heteropairs formed between $\mathbf{1}$ and $\mathbf{3}$ is the slow incorporation rate of $\mathbf{1}$ triphosphate opposite $\mathbf{3}$ in the template and its inefficient extension. These results indicate that the ortho-methoxy substituent in $\mathbf{1}$ in the primer position and the ortho-oxygen atom of $\mathbf{3}$ in the template position do not simultaneously satisfy the demands of efficient synthesis and extension for an ideal unnatural base pair.

In summary, we characterized $\mathbf{3}$ and $\mathbf{4}$, two novel derivatives of the nucleotide analog 2 , which form an interesting unnatural base pair with $\mathbf{1}$. Replacing the ortho-sulfur atom of $\mathbf{2}$ with an oxygen atom disproved self-pair and mispair replications in steady-state experiments, which most limited the replication of the heteropair containing $\mathbf{1}$ and 2 . $^{5}$ However, polar recognition by Kf of the heteropairs between $\mathbf{1}$ and $\mathbf{3}$ was observed; the insertion of $\mathbf{1}$ triphosphate opposite $\mathbf{3}$ and its extension were significantly less efficient than the extension of the heteropair formed by the insertion of $\mathbf{3}$ triphosphate opposite $\mathbf{1}$. These results help to identify the determinants of efficient replication and should contribute to efforts to develop replicable unnatural base pairs by optimizing packing interactions.

\section{Experimental Section}

Synthesis of oligonucleotides. All phosphoramidites were synthesized as described. ${ }^{5 a}$ DNAs were prepared using the $\beta$ cyanoethylphosphoramidite method on controlled pore glass supports $(1 \mu \mathrm{mol})$ with a POLYGEN Professional 12-Column DNA synthesizer and standard methods. ${ }^{8-10}$ After automated synthesis, the oligonucleotides were cleaved from the support by concentrated aqueous ammonia for $1 \mathrm{~h}$ at room temperature, deprotected by heating at $55{ }^{\circ} \mathrm{C}$ for $12 \mathrm{~h}$, and purified by denaturing polyacrylamide gel electrophoresis (12 - 20\%, $8 \mathrm{M}$ urea). The primer oligonucleotides containing unnatural bases at the 3'-end were obtained using 3'-phosphate CPG, which was treated with alkaline phosphatase after deprotection. The oligonucleotides were purified by PAGE, visualized by UV shadowing, and recovered by electroelution. After ethanol precipitation, the concentration of oligonucleotides was determined by UVvis absorption.

Steady-state kinetics. Primer was 5 radiolabeled with $\left[\gamma_{-}{ }^{33} \mathrm{P}\right]$ ATP (GE Healthcare) and T4 polynucleotide kinase (New England Biolabs). Primer-template duplexes were annealed in the reaction buffer by heating to $90{ }^{\circ} \mathrm{C}$ and slow cooling to room temperature. The unnatural nucleobases were evaluated as substrates for $\mathrm{Kf}$ (GE Healthcare) by measuring initial rates at which a $\left[\gamma-{ }^{33} \mathrm{P}\right]$-labeled primer-template, $5^{\prime}$-d(TAATACGAC TCACTATAGGGAGAX) annealed to the 45-mer template, 5'-d(CGCTAGGACGGCATTGGATCGYTCTCCCTATAG TGAGTCGTATTA), was extended with varying concentrations of natural or unnatural nucleoside triphosphates. Each reaction included $40 \mathrm{nM}$ primer template, 0.2 - $1.2 \mathrm{nM}$ enzyme, $50 \mathrm{mM}$ Tris-HCl, pH 7.5, $10 \mathrm{mM} \mathrm{MgCl} 2,1 \mathrm{mM}$ DTT, and $50 \mu \mathrm{g} / \mathrm{mL}$ acetylated BSA. The reactions were initiated by adding a $5 \mu \mathrm{L}$ $2 \mathrm{X}$ dNTP solution to a $5 \mu \mathrm{L}$ solution containing the polymerase and primer-template and incubating at $25^{\circ} \mathrm{C}$ for $3-10 \mathrm{~min}$, and were then quenched with the addition of $20 \mu \mathrm{L}$ of loading dye (95\% formamide, $20 \mathrm{mM}$ EDTA, and sufficient amounts of bromophenol blue and xylene cyanole). The reactions were analyzed by polyacrylamide gel electrophoresis and a Phosphorimager (Molecular Dynamics) was used to quantify gel band intensities corresponding to the extended and unextended primer. The measured velocities were plotted against the concentration of dNTP and fit to the Michaelis-Menten equation to determine $V_{\max }$ and $K_{\mathrm{M}}$. $k_{\text {cat }}$ was determined from $V_{\max }$ by normalizing by the total enzyme concentration.

Acknowledgments. This research was supported by Kyungpook National University Research Fund, 2009.

\section{References}

1. Kornberg, A.; Baker, T. A. DNA replication, 2nd ed.; W. H. Freeman \& Co.: New York, 1992.

2. Piccirilli, J. A.; Krauch, T.; Moroney, S. E.; Benner, S. A. Nature 1990, 343, 33-37.

3. Morales, J. C.; Kool, E. T. Nat. Struct. Biol. 1998, 5, 950-954.

4. Hirao, I.; Kimoto, M.; Mitsui, T.; Fujiwara, T.; Kawai, R.; Sato, A.; Harada, Y.; Yokoyama, S. Nat. Methods 2006, 3, 729-735, and references cited therein.

5. (a) Leconte, A. M.; Hwang, G. T.; Matsuda, S.; Capek, P.; Hari, Y.; Romesberg, F. E. J. Am. Chem. Soc. 2008, 130, 2336-2343. (b) Hwang, G. T.; Romesberg, F. E. J. Am. Chem. Soc. 2008, 130, 14872-14882.

6. McCain, M. D.; Meyer, A. S.; Schultz, S. S.; Glekas, A.; Spratt, T. E. Biochemistry 2005, 44, 5647-5659.

7. Beese, L. S.; Derbyshire, V.; Steitz, T. A. Science 1993, 260, 352355.

8. Beaucage, S.; Iyer, R. Tetrahedron 1992, 48, 2223-2311.

9. Reese, C. B. Org. Biomol. Chem. 2005, 3, 3851-3868.

10. Bang, E.-K.; Kim, S. J.; Kim, B. H. Bull. Korean Chem. Soc. 2004, $25,1619-1620$ 